\title{
The Neolithic Demographic Transition and its Consequences
}

\author{
Edited by Jean-Pierre Bocquet-Appel and \\ Ofer Bar-Yosef \\ Springer Science + Business Media, New York, 2008 \\ ISBN 978-1-4020-8538-3; e-ISBN 978-1-4020-8539-0 \\ U.S. $\$ 319.00,542$ pages
}

\section{Reviewed by}

Helena Fracchia

University of Alberta

Edmonton, Alberta, Canada

The results of a conference held at Harvard in 2006, the collected papers address the demographic process associated with the transition from a hunter-gatherer economy to an agricultural economy in the Neolithic. This newly identified process is characterized by a dramatic increase in birth rate and a concomitant population explosion: the sequence is known as the Neolithic Demographic Transition (henceforth, NDT) and has been detected across Europe, North America, Mesoamerica and South America. The NDT is based on mortality rates, usually from cemetery data, and the methodological innovation of a relative rather than absolute chronology, thus allowing for a more global evaluation of the phenomenon.

The relative chronology is based on the local date of the transition to agriculture. In brief, the NDT was caused by an increase in sedentism which in turn had a significant impact on female fertility and an increase in food supply due to the agropastoral economy. The primary factor of demographic growth is biological reproduction that the authors contend pre-dated the emergence of farming communities.

The nineteen papers are organized into four sections. Part 1 presents the demographic and economic dimensions of NDT, papers in Part 2 address settlement and village practices, Part 3 collects studies dedicated to community size and social organization, Part 4 brings the studies to a global level with discussions of population growth and health in the Neolithic.

Without exception, the papers are stimulating, informative and address a myriad of topics associated with population growth, providing food for thought for historians, archaeologists and anthropologists as well as demographers. The topics discussed include the signature of the NDT in light of the local chronologies, pottery changes, diet transition, architectural innovation, storage 
increasing sedentism, the use of human and animal figurines and questions associated with biological changes and stress.

Inconsistencies in the documentation in the papers on demographic and economic dimensions of the NDT create some difficulty in evaluating the global validity of hypotheses or conclusions by the individual authors. Although the evidence for the NDT hypotheses derives from burials, not all authors provide their sample size nor do they discuss the burial circumstances. Often there is no reference to the total size of the cemeteries or to the percentage of burials actually examined or the location of the burials in the cemetery/in structures. The reader cannot tell if only one portion of the cemetery was examined and therefore the interpretation is not fully documented. In fact, although the NDT is presented as a global phenomenon, serious problems are evident in geographical areas where there is not enough skeletal material to reach a consensus. Equally serious problems exist in using some of the cultural entities as criteria in certain countries. M. Ozdogan ( $A n$ Alternative Approach in Tracing Changes in Demographic Composition: 139-178) questions the validity of using slightly more than 1000 burials to trace the NDT over 5000 years and across a vast geography, particularly in areas like Turkey. Nor is it inconsequential that most of the human burials are not from cemeteries, at least in Turkey, but rather from collective burials within structures: the rationale for the distinction in burials remains unknown. Clearly some distinction between those people buried in structures and those found in cemeteries existed: can we treat these burials equally? Caution should be exercised in making a level playing field without sufficient evidence or without sufficiently documenting the evidence. Early Neolithic burials from hundreds of sites in Bulgaria are less than 100 - was there total recovery of all the skeletal material and from what contexts? The claims, then, for detecting the NDT on a global scale must be supported by providing more complete documentation since NDT is a phenomenon essentially about changing birth rates.

On the other hand, the documentation is complete in other articles, in particular, E. Guerrero et al. (The Nature and Timing of the Neolithic Demographic Transition in the Levant: 57-80). Equally important in establishing the validity of the NDT is the complete analysis of skeletal material so that the proposed demographic explosion, and cultural changes can be tied to concomitant biological changes (I. Hershkovitz and A. Gopher, Demographic, Biological and Cultural Aspects of the Neolithic Revolution: 441-479).

Although it is now widely accepted that sedentism and an improved control of plant resources were major factors in NDT (I. Kuijt, Demography and Storage Systems: 287-313), despite the use of a relative chronology, both the timing and early manifestations of NDT cannot be generalized. For example, villages experiencing a 
rapid growth of the NDT are very different from villages with slower growth rates in North and South America (M. Bandy, Global Patterns of Village Development: 333-357), the NDT in the southern Levant is markedly different from the progression seen in Europe (Kuijt: 309), and these are not isolated cases. While the basic premise is no doubt valid, greater attention needs to be given to various regions, sub-regions and to details before the phenomenon can be generalized. It is not sufficient to introduce relative chronology into the equation. Furthermore, the NDT may not have been as exuberant, there may be numerous reasons for increased fertility and the increase in mortality may have occurred earlier than originally thought. Serious difficulties arise in reconciling the NDT with apparent declining health and high fertility (M. N. Cohen, Implications of NDT for World Wide Health and Mortality in PreHistory: 481-500).

Although the book is certainly comprehensible to the professional, the papers are not intended for the lay reader. The actual reasons for the emergence of sedentism are not discussed by any of the papers: as sedentism is the primary reason for the demographic explosion presented in the NDT, the context for the process could have been presented in the introduction (J.-P. BocquetAppel and O. Bar Yoself, Prehistoric Demography in a Time of Globalization: 1-10). Is it necessary to create the word sedentism when sedentarism exists in English and follows the Latin derivation? On occasion, abbreviations are used without immediate resolution, sometimes leaving the reader in a quandary: for example, the abbreviation LBK is first used on p. 15 and finally defined as referring to the Linear pottery culture on page 208.

Despite the concerns of inadequate and/or inconsistent documentation, the conference proceedings provide an extremely stimulating discussion of the implications of the NDT and its consequences, with all due caution and a number of flags raised about a precocious global application. 